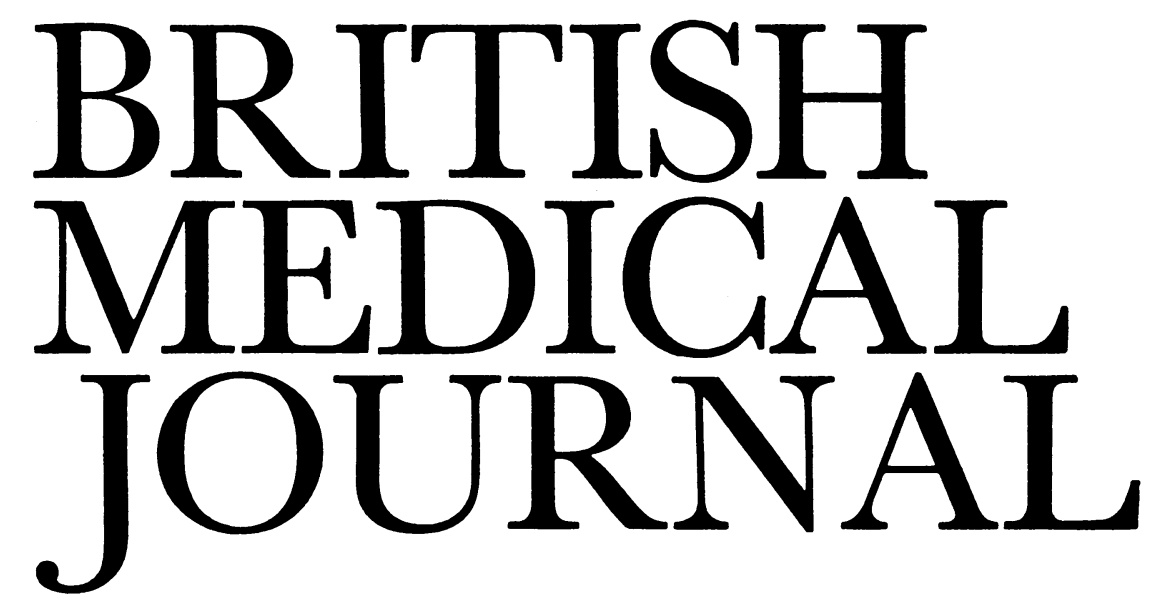

LONDON, SATURDAY 26 JULY 1986

\title{
Did the drug do it?
}

The refined techniques now used in the development of drugs have increased the precision with which individual pharmacological systems may be stimulated or suppressed while closely similar systems serving different functions are more or less unaffected. These advances are usually described in pharmacological jargon: we are now all familiar with drugs which affect mainly $\mathrm{H}_{2}$ but not $\mathrm{H}_{1}, \mathrm{M}_{2}$ but not $M_{1}$, and $\beta_{2}$ but not $\beta_{1}$ receptors. Some important but not immediately obvious consequences have come from these developments-and these may both alter and diminish our chances of detecting adverse drug effects.

The close control of molecular development and then of pharmacological and toxicological testing may have reduced the chances of a drug being released for general use which has predictable adverse effects arising from its mode of action. These changes have also reduced the chances of a drug having unpredictable effects which occur with reasonable frequency during short term use. When side effects are both unpredictable and rare their detection has always been a matter of chance; and such reactions seem likely to continue to pose problems. If modern drugs are perceived as powerful but relatively safe they will be widely used, and in these circumstances toxic reactions of low frequency will become increasingly important. The unavoidable reality is that if prescription numbers are in millions but reactions occur once every 10000 treatments then reactions will occur in hundreds. Paradoxically, however, the individual general practitioner who prescribes may not expect to meet such a reaction even once in 10 years.

Once a drug has been released after clinical trials any adverse effects may come to light in various ways. Individual doctors may report their suspicions through the national yellow card scheme and through reporting cases in medical journals or to the pharmaceutical companies responsible for marketing the products. In all these circumstances the association of event with drug implies a causal link, but this may not necessarily be the case. In each case many questions need answering: might, say, the observed jaundice have been due to non-A non-B hepatitis and not the drug? Might another agent have been responsible? Might the event have been a manifestation of an existing disease? Case reports in medical journals may achieve wide publicity which is not always justified, and editors and reviewers as well as aspiring authors need to maintain critical minds.

Voluntary reporting on yellow cards has now been with us for 20 years and has been a qualified success. It has been particularly useful in detecting unexpected rare adverse effects such as agranulocytosis or jaundice during the course of ordinary illness. In these circumstances the fact that reports are voluntary - and hence are a biased sample of a total of unknown size-may not constitute a barrier to the recognition of the adverse effect. Nevertheless, the picture would be clearer if doctors were naturally more inclined to report and so provide a larger (and probably more representative) selection of reports. Professor Stuart Walker and his colleagues have recently reported the outcome of two studies in which adverse reaction reporting was examined in general practice. ${ }^{12}$ The papers make an interesting pair. Only $15 \%$ of doctors thought that the yellow card scheme was good and needed no improvement, and virtually all the others thought that improvements were necessary; yet no clear recommendations for specific improvements were made by the $\mathbf{4 0 2}$ doctors interviewed.

At least part of the problem seemed to rest with doctors themselves. During the second study, of 36470 consultations, a suspected adverse drug reaction was a factor in 638 . Only in 35 was a report actually made to the Committee on Safety of Medicines. Ten severe adverse reactions were recorded but only two were reported; and of 27 reactions to drugs for which the committee had requested special reporting only three were actually reported. Possibly the circumstances of the study, with reporting of suspected adverse drug reactions on special forms (not yellow cards), may have inhibited formal reporting, but I suspect not. The reason most commonly given for failure to report by doctors was that the suspected reaction was well known, but this reasoning cannot apply to drugs where reporting is specifically requested.

Better feedback to general practitioners about their own reports (as well as through the Committee on Safety of Medicine's Current Problems series) might help to increase the number of reports, as might paying a fee per report, but such changes would still not deal with the fundamental difficulties that reports are voluntary and constitute a biased sample with the extent and nature of bias not clear. And great difficulties remain in attributing causes, particularly when suspected reactions are similar in type to ordinary disease.

At least two other approaches are possible: formal postmarketing surveillance and its offspring event monitoring; and case-control comparisons. At present we have an abrupt transition from closely scrutinised controlled trials in which drug efficacy is assessed in carefully selected individuals to 
free prescription for individuals of all ages with a variety of coincident illnesses and drug treatments. Limited release has been suggested with, say, 10000 treatments being allowed under close scrutiny, but whether this would offer advantages over other varieties of postmarketing surveillance is not clear. Prescription event monitoring has been pioneered in Southampton by Inman and his colleagues. ${ }^{3}$ They have shown that large numbers of reports may be collected from cooperating practitioners; thus over 62000 reports out of some 100000 sought concerning five anti-inflammatory drugs were collected in a recent investigation. The interpretation of these reports has not always been easy. Thus an association between the use of benoxaprofen and photosensitivity and oncholysis was demonstrable, but there were problems, as with other methods, in deciding if ordinary disease was turning up with greater than expected frequency. Inman and his colleagues have compared event rates with and later without treatment in attempting to deal with this problem, but such sequential comparisons may not produce strictly comparable data. Patients with chronic disease may stop one drug but start another, and they often have coincident but unrelated disease. The problem raised by confounding because of associated disease was illustrated during the surveillance of 10000 patients taking cimetidine who, by comparison with non-dyspeptic population controls, were about twice as likely to die during the year after prescription of the drug. They were also more likely to attend hospital with other diseases which were clearly coincidental. ${ }^{4}$ Considerable difficulty arose in disentangling what was coincidental from what might have been caused by treatment.

Retrospective case-control studies are attractive because they offer the opportunity to compare antecedent drug intake in cases with a set disease and in controls. Such studies, however, demand a pre-existing hypothesis, derived, for instance, from yellow card reports, and they take time. They have also been criticised because they may not necessarily cope with all issues of confounding. Nevertheless, casecontrol studies did identify the association between venous thromboembolism and oral contraceptive use, ${ }^{5}$ and they can generate figures which allow us to estimate the increased risk of takers compared with non-takers. We have lately used the method to suggest that though the risk of peptic ulcer bleeding with anti-inflammatory drugs may not appear large per 1000 prescriptions - and may not even be identifiable in postmarketing surveillance-the risk may nevertheless be substantial when seen in the context of the 20 million prescriptions a year that are currently issued in Britain. ${ }^{6}$

The general issues of adverse reaction surveillance have recently been considered by the Committee on Safety of Medicine's Grahame-Smith working party. ${ }^{7}$ This accepted that the yellow card scheme cannot meet all the committee's requirements and recommends that postmarketing surveillance studies should be carried out on most new drugs, particularly when widespread long term use is expected. The working party saw difficulty continuing in detecting rare or long latency reactions and pointed to the possible benefits of record linkage schemes. Problems also arise when new and old drugs are compared, and ad hoc studies seem to be required, particularly as yellow card reports and event monitoring do not seem ideally suited to the purpose.

Determining the risks of drug induced disease does not get easier; indeed, it is getting progressively more costly and taking more time and effort. The public very reasonably wants safer medicines and expects close scrutiny of those on the market. The methods of investigation are, however, only partially effective. If new approaches are to be tried we shall need to examine them critically and to decide whether they are cosmetic or effective.

Professor of Therapeutics,

M J S LANGMAN

University Hospital,

Nottingham NG7 2UH

1 Walker SR, Lamley CE. The attitudes of general practitioners to monitoring and reporting adverse drug reactions. Pharmaceutical Medicine 1986;1:195-203.

2 Lumley CE, Walker SR, Hall GC, Staunton N, Grob PR. The under-reporting of adverse drug reactions seen in general practice. Pharmaceutical Medicine 1986;1:205-12.

3 Inman WHW. Non-steroidal anti-inflammatory drugs. PEM News 1984;2:4-10

4 Colin-Jones DG, Langman MJS, Lawson DH, Vessey MP. Post marketing surveillance of the safety of cimetidine: twelve month morbidity report. Q7 Med 1985;54:253-68.

5 Vessey MP, Doll R. Investigation of relation between use of oral contraceptives and thromboembolic disease. BrMed f 1968;ii:199-205.

6 Somerville KW, Faulkner G, Langman MJS. Non-steroidal anti-inflammatory drugs and bleeding peptic ulcer. Lancet 1986; i:462-4.

Committee on Safety of Medicines. Adverse Reactions Working Party. Second Report. London: HMSO, 1986. (Grahame-Smith report.)

\section{The leaking labyrinth}

In the inner ear the perilymph and endolymph are separated by extremely delicate membranes. Changes in the pressure of the cerebrospinal fluid are transmitted to the perilymph compartment through the cochlear aqueduct. ${ }^{1}$ Pressure changes in the middle ear-such as occur during barotrauma - are also transmitted to the inner ear. If these changes in pressure are excessive the membranes in the inner ear may rupture, and perilymph may then leak into the middle ear through tears at the round or oval window. Tears may also occur inside the labyrinth, mixing perilymph with endolymph and injuring the sensory epithelium. ${ }^{2}$

The pattern of auditory and vestibular symptoms produced by such ruptures is variable. In some series loss of hearing has been the outstanding symptom, ${ }^{3}$ while in others vestibular symptoms have predominated. ${ }^{4}$ The hearing loss is sensorineural, often of sudden onset, and need not be accompanied by vertigo. ${ }^{3}$ The vestibular symptoms may cause ataxia, episodic vertigo, or a sensation of unsteadiness or lightheadedness. ${ }^{4}$ Typically the symptoms develop after physical exertion or a change in pressure-such as after diving or flying. Coughing, sneezing, lifting, straining, or parturition may also cause these injuries. Not surprisingly, leaks may result from head injury and penetrating injuries of the ear. In at least one third of cases, however, the onset is apparently spontaneous. ${ }^{3}$

Undue emphasis has been placed on the "fistula test" in the diagnosis of perilymph leaks. The test is performed by inducing positive and negative pressure in the external ear canal by pneumatic otoscopy or by firm digital pressure on the tragal cartilage. The test result is positive if either nystagmus or a sensation of disequilibrium occurs. In one study the test was positive in only six of 17 patients with surgically proved fistulas, and it gave false positive results in five patients out of $10 .{ }^{5}$ Indeed, there are no specific auditory or vestibular investigations that will confirm or exclude the presence of a perilymph leak. ${ }^{2}$ Diagnosis is heavily dependent on clinical suspicion; it may be made with certainty only when the middle ear is explored surgically.

The approach to treatment of these fistulas must be tempered by the knowledge that many probably heal spontaneously. ${ }^{2}$ When a clinical diagnosis is made the patient 